\title{
Reliability Analysis of Hydrodynamic System for Robot Configuration
}

\author{
HUI LIU* \\ Henan Institute of Economics and Trade, Zhengzhou 450046, CHINA
}

\begin{abstract}
The failure tree and J-M model method are lack of analysis of the importance of each component model, which leads to the low reliability of the analysis results. In view of this problem, a Monte Carlo method based on the shape of the English long-distance robot is proposed. In view of the configuration of the robot, the realization process of the robot shape fluid dynamics system is analyzed. The frequency of accident is determined by Monte Carlo simulation, which is used as the reliability index of the system. In MATLAB, the reliability of the shape fluid dynamic system of robot is analyzed by Monte Carlo method. The system importance name and parameters are determined. The parameter conforms to the statistical function of random variables of each corresponding probability distribution function. According to the parameters, the function of the structure is established. The system is divided into reliable state, failure state and limit state with 0 as the dividing point, and the actual failure probability of the system is calculated. The numerical solution of log domain is simulated by the method of statistical calculation of random variables, and the actual failure probability is expressed by normal distribution function. The experimental results show that the actual failure probability of the method is lower than 5\% under any working load, and the reliability of the analysis results is high.
\end{abstract}

Key words: robot; shape fluid dynamic system; reliability; importance; failure probability

Received: April 13, 2021. Revised: September 15, 2021. Accepted: September 23, 2021. Published: November 29, 2021.

\section{Introduction}

Robot can promote the effect of language courses. Domestic robot design generally pays more attention to the research of robot performance index ${ }^{[1]}$, but ignores the prediction of its reliability, which makes the robot unreliable in the use process ${ }^{[2]}$. It can be seen that the research on the reliability of robot is a very important development direction. However, if the reliability of robot system is completely attributed to the reliability of components and the level of manufacturing technology, and the effect of system design on reliability is not considered, this method is not very perfect. In the case of increasingly complex robot system, it can not solve its reliability problem well. At present, the fault tree model is applied to the reliability analysis of the fluid power system of robot. The fault tree method comes from the design of hardware. Its principle is to refine the software errors according to the tree structure until the cause of the error is found. Although this method can be used in the design phase, it lacks detailed research in the implementation phase. Using the J-M model, it is assumed that the number of errors inherent in the software is an unknown constant, that the errors in the software are independent of each other, that the probability of system failure caused by each error is roughly the same, and that the time interval between failures is also independent of each other. All the errors detected in the test are eliminated, and only one error is excluded at a time. Although the elimination time can be ignored, new errors are constantly introduced in the process of debugging, resulting in unsatisfactory analysis results. In view of this situation, a Monte Carlo method is proposed to analyze the reliability of the fluid dynamic system of the robot.

\section{Analysis of fluid dynamic system for}

\section{the shape of robot}

In the environment, users and robots constitute a 
human-computer interaction system, and the interaction goal is defined as the transmission of English content. The way to achieve this goal is voice dialogue. The behaviors involved in the dialogue between robot and users include listening, speaking and body expression. Make judgment on the feedback of users and plan the next step behavior to form a closed-loop system until the users actively interrupt the interaction (the robot will enter the sleep state until it is awakened again) ${ }^{[3]}$.

Robot is a tool for signal reception, preprocessing, distribution and result display. The robot directly receives the users' speech signals, and uses the speech recognition function inside the robot to convert the speech signals into text signals that can be understood by the program. According to the input signal, call the corresponding server interface to achieve different functions. When the server returns the result of interface call, the result is displayed to the users by using the voice synthesis function and body action function inside the robot ${ }^{[4-6]}$. The configuration of robot is shown in Figure 1.

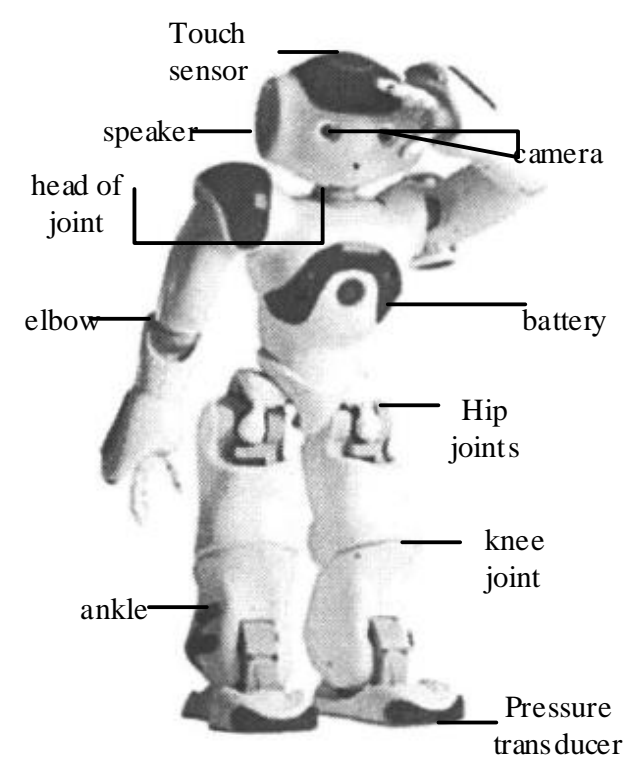

Figure 1 Configuration of robot

The realization of speech function of robot mainly depends on the running environment and server environment of robot. In the environment of robot, Python language is used to realize the basic processing of speech signal and interface call function, using Python version $2.7^{[7-9]}$. In the server environment, Java language is used to realize the core function of robot, including the different feedback of different quality word pronunciation in the word practice function. In the role-playing dialogue, the role switching of users in the dialogue, the feedback of users' different speech content in the free dialogue function, etc. The server program adopts springboot framework, and the program is published on nginx web server ${ }^{[10-12]}$. robot and server program communicate through restful style interface. robot and server are in the same $\operatorname{LAN}^{[13,14]}$.

For the shape fluid design of robot, the topological information of the optimal shape is often unknown before optimization, which leads to the traditional shape optimization method is no longer effective. Topology optimization is to optimize the size, shape and topological structure of the optimization target at the same time. It is a more advanced optimization method than ordinary shape optimization. It can guide the direction of optimization by setting constraints and objective functions, so that researchers can get rid of the problems of empirical design ${ }^{[15]}$. The flow chart of the robot shape fluid dynamic system is shown in Figure 2.

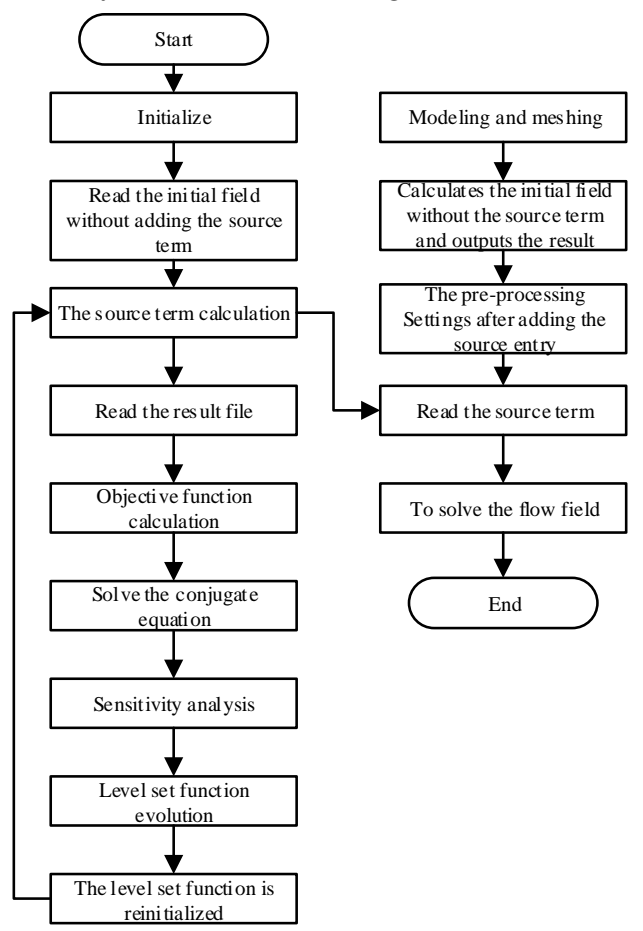

Figure 2 Flow chart of robot shape fluid dynamic

$$
\text { system }
$$

The fluid dynamic system of robot shape mainly depends on three parts. (1) MATLAB calls CFX solver to simulate fluid automatically. (2) The dynamic link library is established to enable applications to call functions that do not belong to their executable code, which solves the 
problem that Fortran programs cannot be directly recognized by $\mathrm{CFX}$, and the source information can be smoothly transferred to CFX. (3) MATLAB calls CFX post-processing module to export the calculation results in the specified format and path for MATLAB to read and carry out subsequent calculation ${ }^{[16]}$. The invention combines two commonly used softwares MATLAB and CFX, MATLAB controls the topology optimization algorithm, CFX solves the flow field, establishes an interface, and creates a joint solution platform, so that the two can call each other and transfer data. Taking two advantages of each software can not only solve the multi-objective optimization, but also greatly reduce the optimization time ${ }^{[17]}$.

\section{Reliability analysis of fluid dynamic}

\section{system for robot configuration}

Monte Carlo method is a reliability calculation method based on the principle of mathematical statistics, which is also called statistical experiment method or random simulation method. Its basic principle is to establish a probability model according to the problem, get the sample through some kind of false experiment with numbers, then carry out statistical processing, and take the result as the solution of the problem.

\subsection{Simulation method based on Monte Carlo}

The basic principle of Monte Carlo method is as follows: let $q_{1}, q_{2}, \ldots, q_{n}$ be $n$ independent variables with the same distribution law, and $q$ and $p^{2}$ represent the same finite mean value and variance respectively, then any $\phi>0$ has:

$$
\lim _{n \rightarrow \infty} W\left\{\left|\frac{1}{n}\right| \sum_{i=1}^{n} \geq \varphi\right\}=0
$$

If $R$ is set as the failure event of the fluid power system of the robot, the probability of the event is $P(R)$. After $\mathrm{n}$ tests, the frequency of fault events $R$ in the fluid dynamic system of the robot shape in English distance is $\mathrm{m}$, and the frequency of the accidents is obtained as follows:

$$
P(R)=\frac{m}{n} \times 100 \%
$$

According to the Formula (2), for any $\phi>0$, there are:

$$
\lim _{n \rightarrow \infty}\left\{\left\{\left|\frac{m}{n}-P(R)\right|<\phi\right\}=1\right.
$$

The application of Monte Carlo method is as follows: in this paper, the reliability of fluid power system which affects the shape of robot is analyzed by random stratified sampling. Random stratified sampling is to divide the population into several subpopulations according to one or several characteristics, and each subpopulation is called a layer. Then a sub sample is randomly selected from each layer, and these sub samples together are the total sample. The sample number of each layer is determined by non proportional distribution method. When the proportion of the number of cases in a certain level is too small, the proportion of the number of cases in the total sample can be increased artificially in order to make the characteristics of that level adequately reflected in the sample. But doing so increases the complexity of inference. In general, the variables that are used for stratification are hierarchical variables, and the ideal hierarchical variables are the fault events to be detected or the variables related to them.

According to the principle of random sampling, that is, to ensure that each unit in the population has the same chance to be selected, the method of sampling. When inferring the population according to the sample data, one group by one is substituted into the structure to determine whether the failure occurs, so as to obtain the reliability [18, 19].

\subsection{Reliability simulation program block diagram}

According to the statistical function of random variables that obey the corresponding probability distribution function, the $m^{\prime} \times n^{\prime}$ random variable array is generated by using the corresponding random number instruction, and is substituted into the function function one by one to get the result array. Count the number $\mathrm{x}$ of elements greater than 0 in the array to determine the system reliability ${ }^{[20-25]}$. Matlab is a matrix factory, which mainly faces the computing environment of interactive programming. To a great extent, it can get rid of the editing mode of the traditional non interactive programming language. It can process and analyze the 
digital image, has a friendly user interface and is close to the natural language of mathematical expression. It provides a large number of convenient and practical processing tools for simulation research. Therefore, in MATLAB, Monte Carlo method is used to analyze the reliability of the shape fluid dynamic system of robot. Figure 3 is the program block diagram.

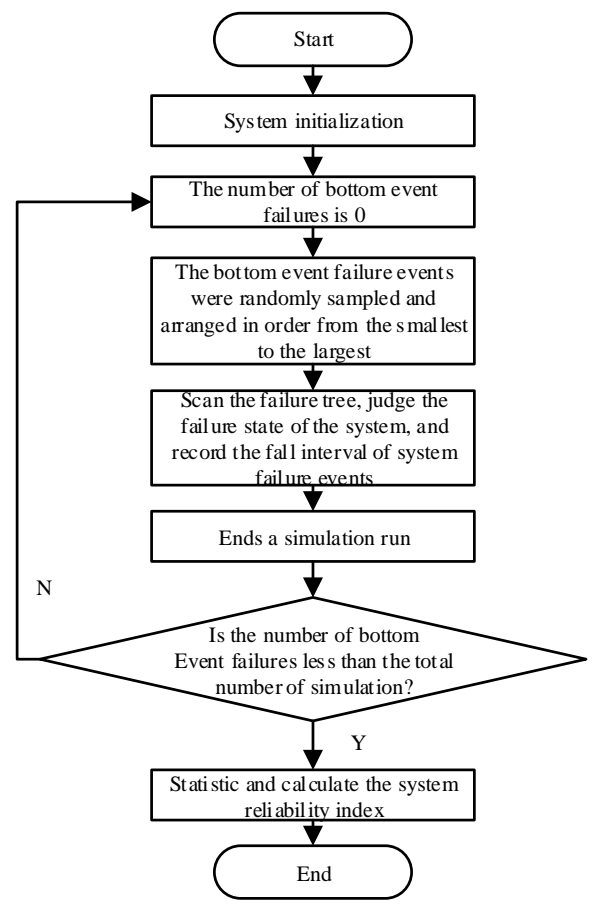

Figure 3 Block diagram of reliability simulation program

According to Figure 3, the relationship between function function and basic random variable is implicit in reliability analysis. From the perspective of reliability calculation, the value of given sample point is analyzed by CAE tool, and then the reliability is calculated by statistics. The following two problems must be solved when the CAE tool is used for reliability simulation calculation:

(1) The reliability calculation program package and call CAE software to realize the calculation of the operating load of the hydrodynamic system with the shape of the robot;

(2) The gradient calculation can be used to determine whether the load is within the specified load range by using the finite difference method on the basis of obtaining the operating load of the hydrodynamic system with the shape of the robot.

After initializing the hydrodynamic system of robot, the initial number of failures is determined to be zero, and then the failure events are randomly selected from the historical database and arranged from the minimum to the maximum probability. When scanning the fault tree, according to the probability of the selected failure event, the time interval of the probability decline after the system failure is judged, so as to end the simulation operation.

\subsection{Actual failure probability analysis}

The purpose of the reliability verification of the fluid power system of the robot is to test whether the fluid power system structure of the robot meets the specified reliability under the specified load. In the reliability analysis, the influencing factors should be considered first, as shown in Table 1.

Table 1 System importance name and parameters

\begin{tabular}{|c|c|c|c|}
\hline Number & $\begin{array}{l}\text { Probability } \\
\text { importance }\end{array}$ & $\begin{array}{c}\text { Mode } \\
\text { importance }\end{array}$ & Event name \\
\hline$\# 1$ & 0.035 & 1.000 & $\begin{array}{l}\text { Power } \\
\text { failure }\end{array}$ \\
\hline$\# 2$ & 0.051 & 1.000 & $\begin{array}{l}\mathrm{X} \text {-axis } \\
\text { motor }\end{array}$ \\
\hline$\# 3$ & 0.052 & 1.000 & $\begin{array}{l}\text { Y-axis } \\
\text { motor }\end{array}$ \\
\hline$\# 4$ & 0.063 & 1.000 & $\begin{array}{l}\text { Z-axis } \\
\text { motor }\end{array}$ \\
\hline$\# 5$ & 0.015 & 1.000 & $\begin{array}{l}\text { Computer } \\
\text { failure }\end{array}$ \\
\hline \#6 & 0.029 & 1.000 & $\begin{array}{c}\text { Control card } \\
\text { hardware }\end{array}$ \\
\hline
\end{tabular}

It can be seen from Table 1 that the mode importance of each component is the same, which indicates that a failure of each component in the system will cause a system failure. The greater the value of probability importance, the greater the probability of failure of the component, which is the weak link of the system. Therefore, according to this table, we can judge the weak links of the system and determine the maintenance sequence when the system fails.

According to Table 1, the structure function is established as follows:

$$
f=g\left(Y_{1}, Y_{2}, Y_{3}, Y_{4}, Y_{5}, Y_{6}\right)
$$

In Formula (4): $Y_{1}, Y_{2}, Y_{3}, Y_{4}, Y_{5}, Y_{6}$ respectively 
represent the power failure, $\mathrm{X}$-axis motor, Y-axis motor, $\mathrm{Z}$-axis motor, computer failure and control card hardware factors that affect the reliability of the fluid power system of the robot; $g(Y)$ represents the function.

The function divides the system into three different states with 0 as the dividing point:

(1) If $f>0$, the system is reliable, which is represented by $P_{1}$;

(2) If $f<0$, the system fails, which is represented by $P_{2}$;

(3) If $f=0$, the system is in the limit state.

The actual failure probability of the fluid dynamic system of the robot is as follows:

$$
P_{2}=1-P_{1}
$$

Using Monte Carlo method and statistical calculation of random variables, the numerical solution in mathematical field is simulated and solved, which is expressed by normal distribution function. Normal distribution, also known as "habitus distribution", is a very important probability distribution. The area between the curve and the horizontal axis is always equal to 1 . This is the probability of integrating the function of probability density from positive infinity to negative infinite function is 1 . That is to say, the sum of frequencies is $100 \%$. The normal distribution function is shown in Figure 4.

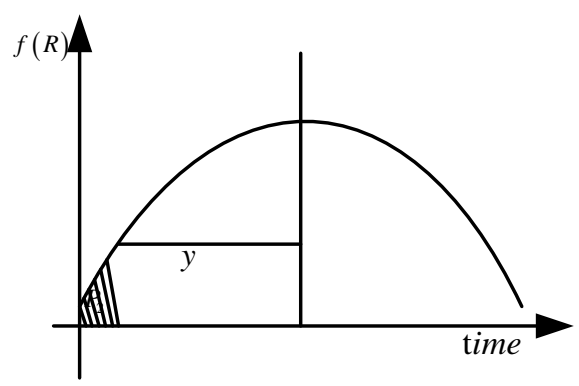

\section{Figure 4 Normal distribution function}

It can be seen from Figure 4 that $y$ is inversely proportional to the actual failure probability. The smaller $y$ is, the higher the actual failure probability is, and the lower the reliability is; on the contrary, the higher.

\section{Experiment}

In order to verify the rationality of the reliability analysis of the fluid power system of the robot, the experimental verification analysis is carried out.

\subsection{Fluid dynamics simulation of robot shape}

Grid is the basis of model construction and expression in CFD simulation, and it is the bearing element of numerical simulation. Its quality plays a decisive role in the accuracy and efficiency of numerical simulation. ANSYS ICEM CFD software is used for mesh generation, which has powerful ability of hexahedral structured mesh generation, and can divide hexahedral mesh in any shape model. Considering the distance between distance learning and the size of air flow space, the air fluid area is set within $50 \mathrm{~m}$ around the robot. Import the fluid model into the software, as shown in Figure 5.

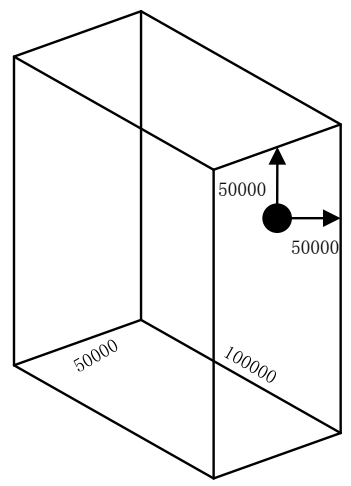

Figure 5 Fluid model

It can be seen from Figure 5 that when meshing, the fluid profile is first outlined in the whole block, and the outer O-Block is established, so as to generate the boundary layer mesh near the wall. The total number of nodes is 4541832 , and the total number of cells is 4658339. The quality of the grid is above 0.3 .

\subsection{Parameter setting}

Boundary conditions refer to the variation of the variables or their first derivatives on the boundary of the solution domain with time and place. The basic boundary conditions include flow inlet and outlet boundary, given pressure boundary, wall boundary, symmetric boundary and periodic boundary. The external flow field of the robot is solved in a steady state, and the boundary conditions are set as shown in Figure 6. 


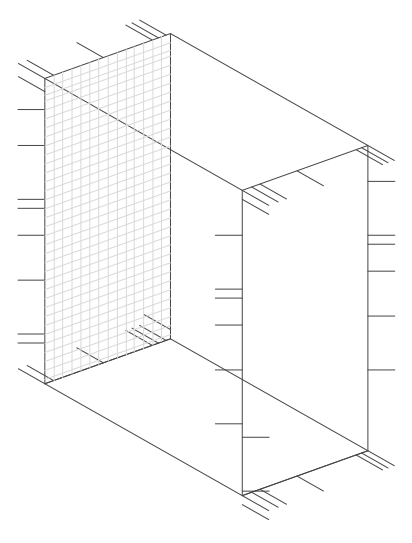

(a) IN

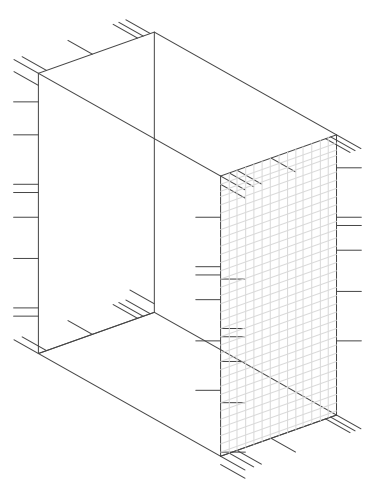

(b) OUT

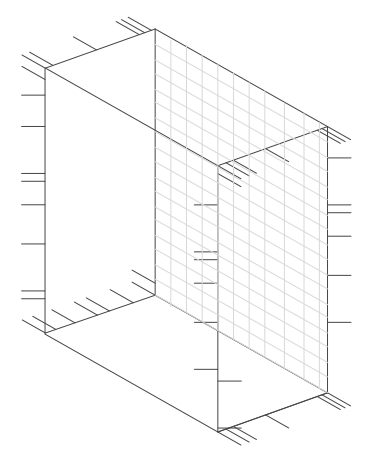

(c) WALL and RO

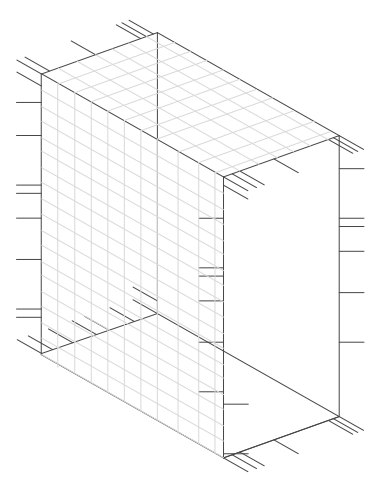

(d) AIR

\section{Figure 6 Boundary condition setting}

As can be seen from Figure 6, the details of the boundary setting of the inlet IN, outlet OUT, bottom WALL, robot RO and AIR are as follows:

(1) Inlet IN

Inlet boundary type is selected as inlet, and the fluid can not be entered or out, and the direction is perpendicular to the surface, and the turbulence intensity is set to medium. As shown in the grid area in Figure 6(a).

(2) Outlet OUT

Outlet boundary type is selected as outlet, and the fluid can not be input, and the static pressure is set to 0.1 $\mathrm{MPa}$. As shown in the grid area in Figure 6(b).

(3) Bottom WALL and robot RO

For the bottom boundary type, the smooth wall boundary condition without sliding is selected as the adiabatic condition. The type of robot boundary is $\mathrm{W}$ all, and the smooth wall boundary condition without sliding is selected. As shown in the grid area in Figure 6(c).

(4) AIR

The air boundary type is Opening, the fluid can enter and exit, the opening pressure is $0.1 \mathrm{MPa}$, and the turbulence intensity is medium. As shown in the grid area in Figure 6(d).

\subsection{Experimental results and analysis}

(1) Normal working load

Under normal working load, the actual failure probability based on fault tree, J-M model and Monte Carlo analysis method is analyzed, as shown in Figure 7.

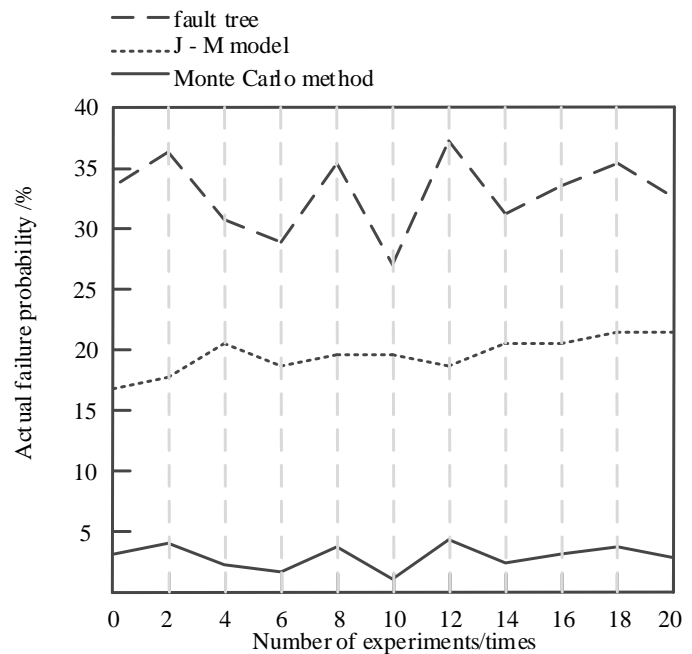

Figure 7 Actual failure probability of three methods under normal working load 
It can be seen from Figure 7 that the actual failure probability of the three methods is less than $40 \%$, and the actual failure probability of the fault tree reliability analysis method is between $20 \%-40 \%$; the reliability analysis method of J-M model is between $15 \%-25 \%$; the actual failure probability of the reliability analysis method based on Monte Carlo method is between 0-5\%. Therefore, the reliability analysis method based on Monte Carlo method is more reliable under normal working load.

(2) Abnormal working overload

Under the abnormal working probability, the actual failure probability of the three methods is analyzed, as shown in Table 2.

Table 2 Actual failure probability of three methods under abnormal working overload

\begin{tabular}{cccc}
\hline Load $/ \mathrm{KN} / \mathrm{m}^{2}$ & Fault tree & $\begin{array}{c}\mathrm{J}-\mathrm{M} \\
\text { model }\end{array}$ & $\begin{array}{c}\text { Monte Carlo } \\
\text { method }\end{array}$ \\
\hline 0.5 & $21 \%$ & $9 \%$ & $1 \%$ \\
1.0 & $37 \%$ & $23 \%$ & $2 \%$ \\
1.2 & $45 \%$ & $42 \%$ & $2 \%$ \\
1.5 & $62 \%$ & $45 \%$ & $5 \%$ \\
2.0 & $76 \%$ & $56 \%$ & $4 \%$ \\
2.5 & $78 \%$ & $65 \%$ & $4 \%$ \\
\hline
\end{tabular}

It can be seen from Table 2 that when the load is $0.5 / \mathrm{kn} / \mathrm{m}^{2}$, the actual failure probability of the three methods is relatively low. As the load increases, the actual failure probability of the three methods increases. When the load is $1.5 / \mathrm{kn} / \mathrm{m}^{2}$, the probability of failure of the three methods is higher; when the load is $2.5 / \mathrm{kn} / \mathrm{m}^{2}$, the probability of failure of the three methods is $78 \%$. The actual failure probability of Monte Carlo method is $5 \%$. Therefore, the reliability of reliability analysis method based on Monte Carlo method is relatively high under abnormal working load.

\section{Conclusion}

According to the working mode of the fluid power system of the robot, the reliability theory is applied to the system reliability analysis. In MATLAB, the Monte Carlo method is used to analyze the reliability of the system, and the relevant data are obtained. The experimental results show that the method has high reliability. The process of Monte Carlo digital simulation actually reflects the essential process of system life development. Through the simulation, the staff can gain insight into how the system failure is caused by the failure of the internal unit of the system, understand the failure mode and weak link of the system, and increase the operation experience and test maintenance experience. This is of special significance for the design, use, detection and maintenance of the system.

The Monte Carlo method provides a feasible strategy and thought in technology for the weak link of robot in the use process or in some specific environment, which has a certain practical significance. Taking this as the future research direction, combined with fault tree, the simulation calculation of fluid power system programming is carried out through computer programming, which provides reference for its reliability prediction.

\section{References}

[1] Giacomo G, Zhu L L, Gallaire F. The Hydrodynamics of a Micro-Rocket Propelled by a Deformable Bubble. Fluids, 2019, 4(1):48.

[2] Suzuki K, Kawabata K. Development of a Robot Simulator for Decommissioning Tasks Utilizing Remotely Operated Robots. Journal of Robotics and Mechatronics, 2020, 32(6):1292-1300.

[3] Chen D, Yang Z, Ji Y, et al. Deformable Ferrofluid-Based Millirobot with High Motion Accuracy and High Output Force. Applied Physics Letters, 2021, 118(13):134101.

[4] Gong L, Li X D, Xu W B, et al. Naturally a Humanoid Tri-Co Robot in a Real-Time Scenario Using First Person View. Science China Information Sciences, 2019, 62(5):17-19.

[5] Feireisl E, Li Y. On Global-In-Time Weak Solutions to the Magnetofluid Dynamic System of Compressible Inviscid Fluids. Nonlinearity, 2020, 33(1):139-155.

[6] Zhu W, Si J, Pang Z, et al. Rapid Deployment and Continuous Shape Maintenance of Tethered-Space Net Robot Based on Single-Pulse Action. Advances in Space Research, 2020, 67(5):1477-1489.

[7] Garcia J, Shafie D. A Humanoid Robot to Walk Faster through Safe Reinforcement Learning. Engineering Applications of Artificial Intelligence, 2020, 88(Feb.):103360. 
[8] Sohn H Y, Roy S. Fluid-Solid Reaction Kinetics for Solids of Nonbasic Geometries: Comparison of the Sohn-Wall Method and the Shape-Factor Method. Industrial and Engineering Chemistry Research, 2020, 59(13):5720-5724.

[9] Costa L, Nascimento T P, Goncalves L. Online Learning and of Emergent Behaviors in Multi-Robot Teams. IEEE Access, 2019, 7: 158989-159001.

[10] Zarth A, Klemens F, Thter G, et al. Towards Shape Optimisation of Fluid Flows Using Lattice Boltzmann Methods and Automatic Differentiation. Computers \& Mathematics with Applications, 2021, 90(1):46-54.

[11] Duraisamy P, Sidharthan R K, Santhanakrishnan M N. Design, Modeling, and Control of Biomimetic Fish Robot: A Review. Journal of Bionic Engineering, 2019, 16(6):967-993.

[12] Costa L, Nascimento T P, Maia R, et al. N-learning: An Approach for Learning and Skills in Multirobot Teams. Robotica, 2019, 38(1):48-68.

[13] Russell D A, Sparrow V. A Master's Degree in Acoustics through Distance Education from Penn State. The Journal of the Acoustical Society of America, 2019, 145(3):1707-1707.

[14] Compare M, Bellani L, Zio E. Optimal Allocation of Prognostics and Health Management Capabilities to Improve the Reliability of a Power Transmission Network. Reliability Engineering \& System Safety, 2019, 184:164-180.

[15] Hughes-Roberts T, Brown D, Standen P, et al. Examining Engagement and Achievement in Users with Individual Needs through Robotic-Based Sessions. British Journal of Educational Technology, 2019, 50(5):2736-2750.

[16] Salmon H, Couraud L, Roblin C, et al. Microrobot-in-Glass for Dynamic Motion Analysis and Wider in Vitro Applications. Micro \& Nano Letters, 2019, 14(8):882-886.

[17] Yao KC, Wu T C, Hsu L C, et al. Evaluating Thematic-Approach of Robot Design and Practice Course through Psychomotor and Affective Domains. ICIC Express Letters, 2019, 13(1):41-49.

[18] Pransky J. The Pransky Interview: Dr Hod Lipson, Professor at Columbia University; Robotics, AI,
Digital Design and Manufacturing Innovator and Entrepreneur. Industrial Robot, 2019, (5):568-572.

[19] Censi A. A Class of Co-Design Problems with Cyclic Constraints and Their Solution. IEEE Robotics and Automation Letters, 2019, 2(1):96-103.

[20] Lane M, Zimmerman J B. Controlling Metal Oxide Nanoparticle Size and Shape with Supercritical Fluid Synthesis. Green Chemistry, 2019, 21(14):3769-3781.

[21] Doyon B. Generalised Hydrodynamics of the Classical Toda System. Journal of Mathematical Physics, 2019, 60(7):073302.

[22] Chaturvedi R, Islam A, Sharma A, et al. Design and Analysis of Mechanical Gripper of Aristo- Robot for Welding//International Conference on Engineering, Technology and Management for the Sustainable Development. 2020, 6(22):2302-2309.

[23] Bhagat R A, Baig S, Naik N, et al. Modeling and Analysis of a Dust Cleaning Robot. Pramana, 2019, 9(1):175-179.

[24] Gaeid K S, Nashee A F, Ahmed I A, et al. Robot Control and Kinematic Analysis with 6DoF Manipulator Using Direct Kinematic Method. Bulletin of Electrical Engineering and Informatics, 2021, 10(1):70-78.

[25] Ferrari F, Divan S, Guerrero C, et al. Human-Robot Interaction Analysis for a Smart Walker for Elderly: The ACANTO Interactive Guidance System. International Journal of Social Robotics, 2020, 12(2):12-16.

\section{Creative Commons Attribution License 4.0 (Attribution 4.0 International, CC BY 4.0)}

This article is published under the terms of the Creative Commons Attribution License 4.0 https://creativecommons.org/licenses/by/4.0/deed.en_US 